\title{
Determinación de distritos de aptitud acuícola mediante la aplicación de sistemas de información geográfica
}

\author{
Claudio Silva G., Rodolfo Olivarí M. y Gabriel Yany G. \\ Escuela de Ciencias del Mar, Universidad Católica de Valparaíso \\ Casilla 1020, Valparaíso, Chile \\ E-mail: carsat@ucv.cl
}

Recibido 30 diciembre 1998; versión corregida 28 octubre 1999; aceptado 29 octubre 1999.

RESUMEN. En este artículo se muestra un procedimiento metodológico para la determinación de distritos aptos para el desarrollo de cultivo de salmónidos y moluscos bivalvos, que incluye distintos procedimientos de análisis cartográfico digital y de análisis matricial de bases de datos relacionales en un Sistema de Información Geográfica (SIG).

La proposición plantea el diseño de un modelo que integra los criterios ambientales con los requerimientos específicos del recurso. Se considera la construcción de sitios a través del procesamiento de imágenes, cálculo de capacidad de carga y la evaluación de la toma de decisión mediante análisis de multi-criterio y multi-objetivo.

Palabras claves: distrito, aptitud, acuícola, SIG, criterios ambientales.

\section{Aquaculture site selection using a geographical information system}

\begin{abstract}
This paper shows a methodological procedure to aquaculture site selection for the development of salmonid and bivalve mollusk culture. The proposal includes different processes of digital cartographic analysis and management of relational data bases in matrixes work applying a Geographical Information System (GIS).

The basis of this procedure is a model design that integrate the environmental criteria with the species requirements. It is considerate the site construction by the processing of images, assessing the carrying capacity and the decision support evaluation through a multi-criteria and multi-objective.
\end{abstract}

Key words: site, selection, aquaculture, GIS, environmental criteria.

\section{INTRODUCCIÓN}

Entre los problemas de la planificación de la expansión de la acuicultura, está valorar con precisión la disponibilidad de recursos hídricos ya que este proceso de toma de decisiones sobre lugares idóneos, exige acopiar y analizar la información necesaria respecto de las condiciones espaciales de los distintos factores de producción (Meaden y Kapetsky, 1992). Los Sistemas de Información Geográfica (SIG) pueden llegar a ser herramientas técnicamente eficientes para realizar estos estudios de valoración de los recursos hídricos dado que poseen ventajas operacionales que permiten almacenar, manejar y analizar una gran cantidad de datos de diversa clase que tienen una base geográfica común.

La inclusión de los SIG en el sector acuícola se considera importante como apoyo al desarrollo y crecimiento de los cultivos marinos, hoy en notable expansión en las comunidades de pescadores artesanales dada la disminución de los bancos natu- rales de especies de alto valor económico tales como ostión, ostra, chorito, loco y erizo. En este contexto, es interesante notar lo que el Banco Mundial recientemente ha dicho, con respecto a implementar acuicultura en pequeña escala: "El apropiado uso de los Sistemas de Información Geográfica y tecnologías de sensores remotos en íntima cooperación con organismos como FAO sería muy deseable" (Meaden y Do Chi, 1996).

Aunque la utilización de nuevas áreas de aguas para cultivo es frecuente en nuestro país, no se realizan estudios tendientes a la evaluación del potencial de cultivo de estos lugares, y con mayor razón son menos los trabajos de investigación apoyados por SIG en este campo.

En este marco, el presente estudio entrega una secuencia metodológica de aplicación de un Sistema de Información Geográfica para la determina- 
ción de distritos de aptitud acuícola. Se sintetiza el método de estudio trabajado y los resultados obtenidos por los autores en un área geográfica del fiordo Aysén, XI Región, Chile.

\section{MATERIALES Y MÉTODOS}

\section{Bases Conceptuales}

El estudio del potencial o aptitud de cultivo de un área geográfica identificada respecto de una especie determinada, no siempre es considerada por los gestores de la planificación de la actividad. Estas evaluaciones consideran un distrito o unidad espacial en la cual el comportamiento de un determinado recurso es homogéneo, es decir, los parámetros o características que lo definen son constantes o varían discretamente.

La aptitud para cultivo es la disposición natural, que en este caso resulta de la integración de las características físicas, químicas y biológicas de un distrito con los requerimientos de la especie objeto de cultivo. Una de las formas de estimar la aptitud (A) de un distrito es determinando el grado de probabilidad de éxito de cultivo, que se puede definir como la sumatoria de la valoración de los criterios de selección $(\mathrm{C})$ por sus respectivos pesos $(\mathrm{P})$ :

$$
\mathrm{A}=\sum_{i=1}^{\mathrm{n}} \mathrm{C}_{i} * \mathrm{P}_{i}
$$

En términos cuantitativos, se puede estimar el potencial de cultivo de un distrito determinando la capacidad de carga de recursos. El diseño metodológico para el cálculo de la capacidad de carga de recursos se desarrolla basándose en la integración de los criterios biológicos relativos a las especies objetivo, oceanográficos y meteorológicos que modifican las condiciones del sitio, y los relativos a la disponibilidad de superficie de éste.

En general, la capacidad de carga (CC) para un cultivo intensivo está dada por el oxígeno disponible (OD), la tasa de consumo de oxígeno por individuo (CO) de talla comercial, la tasa de recambio (R) o tiempo de residencia de las aguas y el área del distrito (AD), y está definida por:

$$
C C=\frac{O D}{C O * R * A D}
$$

\section{PROPOSICIÓN METODOLÓGICA}

\section{Implementación de la base de datos digital}

La implementación del SIG para un estudio de evaluación de acuicultura comienza con la selección de la información necesaria a ingresar al sistema. Se elabora una base de datos digital de los criterios ambientales (temperatura del mar, salinidad, oxígeno disuelto, velocidad y dirección de viento, altura de ola, corrientes, batimetría, topografía, presencia de depredadores y otros) del área geográfica en estudio (Figs. 1 y 2), la generación de layers o planos de información puede tener un origen automatizado (imágenes satelitales) o semiautomatizado si procede de cartografía temática o tipografía convencional e ingresado mediante escáner o procesos de digitalización. La información obtenida de muestreos in situ es digitada y luego completada mediante procesos de interpolación, donde se utilizan programas computacionales específicos para cartografía digital de superficies (por ejemplo SURFER, MATLAB, y otros) utilizando métodos de interpolación (por ejemplo el método geoestadístico Krigging, Vecino Cercano, lineal).

De este modo, considerando los requerimientos técnicos del software SIG utilizado (por ejemplo IDRISI, MAPINFO, y otros), el área en estudio representa una matriz de datos de $\mathrm{X}$ columnas por $\mathrm{Y}$ filas con una resolución espacial (tamaño de pixel) determinada por el número de datos por unidad de medida, sobre esta base se trazan mapas temáticos de cada uno de los factores ambientales relevantes.

Una vez ingresada la información al sistema, comienza la etapa de generación de información complementaria que los SIG permiten (Fig. 2). Ello es posible gracias a las funciones de superposición gráfica, topológica y de base de datos que estos SIG poseen y a su capacidad de entregar un valor $\mathrm{o}$ atributo a cada elemento gráfico y asociar una base de datos. Esta etapa comprende procesos de simulación digital de la realidad ambiental del área de estudio (por ejemplo la simulación de la altura de ola máxima en base a la información espacio-temporal de los vientos máximos).

\section{Construcción de distritos}

En la construcción de sitios se consideran las limitaciones o restricciones de cada uno de los parámetros que regulan los cultivos de las especies acuícolas, que en este caso corresponden a factores ambientales, posteriormente se realiza un proceso 
de intersección booleana entre los planos de información o superposición gráfica de éstos, para generar finalmente un mapa que muestra áreas o distritos para cultivos acuícolas (Figs. 1 y 3). De este modo la superposición gráfica y de atributos de los planos de información, permite generar distritos o unidades homogéneas (polígonos) respecto a los parámetros ambientales. Los polígonos generados tienen asociados la base de datos ambiental, de este modo con ayuda del SIG se pueden obtener los valores de los criterios ambientales (por ejemplo valores mínimo, medio y máximo de temperatura superficial, oxígeno disuelto, batimetría, etc.) para cada polígono o distrito construido.

Por ejemplo, para el área geográfica estudiada del fiordo Aysén, basándose en las limitaciones máximas y mínimas de los parámetros que regulan los cultivos de salmónidos en balsas-jaulas, se obtuvo que los factores que restringen la actividad son la batimetría, salinidad y altura de la ola. Los demás parámetros se encuentran dentro de los rangos de tolerancia de las especies. Mediante los procesos de superposición gráfica con el SIG se obtienen 3 distritos aptos con distintas coberturas espaciales (Fig. 3).

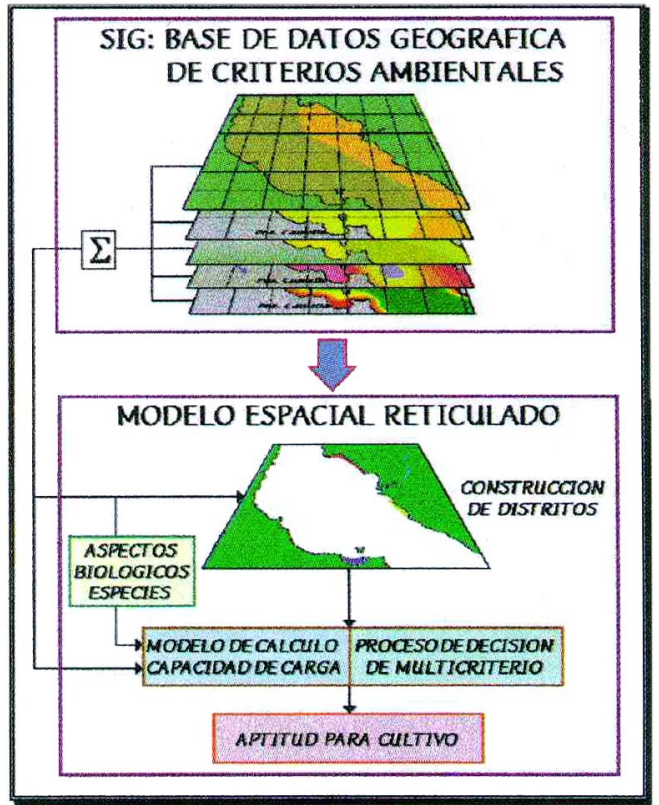

Figura 1. Método propuesto para la determinación de distritos de aptitud.

Figure 1. Proposed Method to aquaculture site selection.

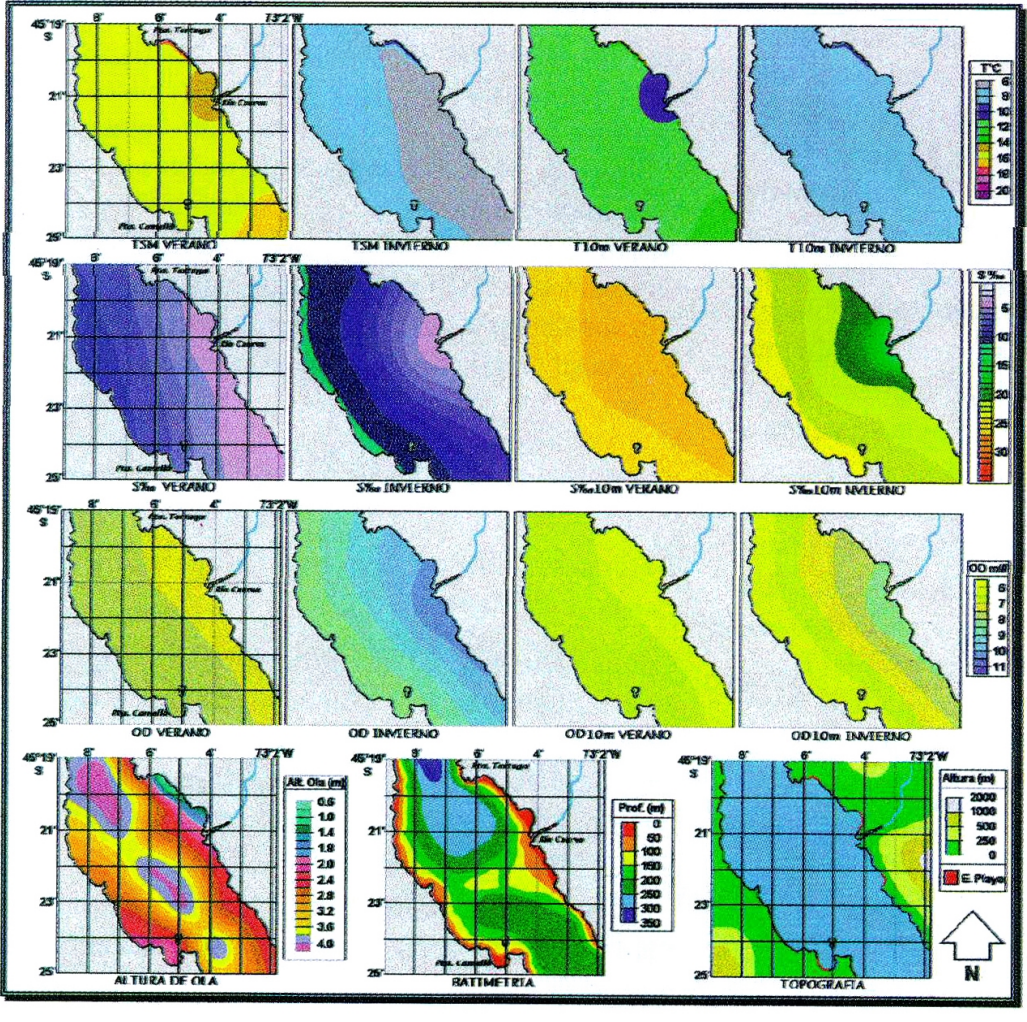

Figura 2. Distribución espacial de los criterios ambientales utilizados en la determinación de distritos de aptitud acuícola.

Figure 2. Environmental criteria distribution used in the aquaculture site selection. 


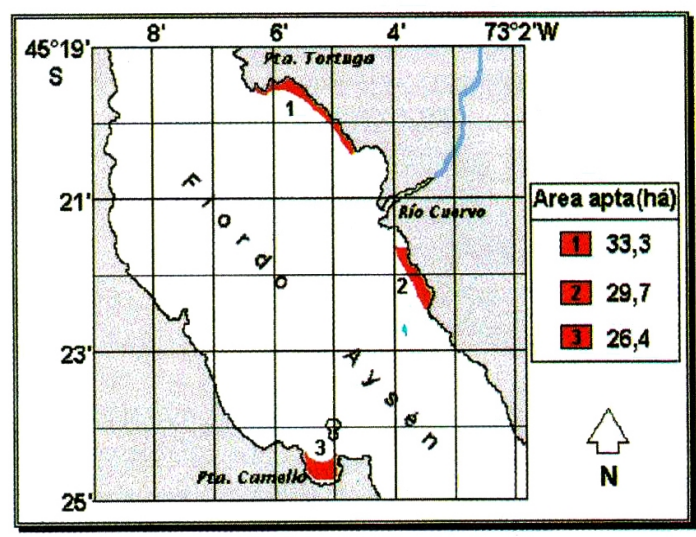

Figura 3. Mapa que muestra distritos para cultivo de salmónidos en balsas-jaulas obtenidos del análisis con el SIG IDRISI.

Figure 3. Locations and surface areas for the development of salmonid cage culture.

\section{Capacidad de carga}

El cálculo de la capacidad de carga de recursos se desarrolla basado en la integración de los criterios biológicos relativos a las especies objetivo, oceanográficos y meteorológicos que modifican las condiciones del fiordo o bahía, y los relativos a la disponibilidad de superficie en cada sitio. El método considera el oxígeno disponible como variable de vital importancia tanto para el cultivo de salmónidos como para moluscos bivalvos, ya que esta variable tiene un rol fundamental en los procesos biológicos de los recursos vivos. Se establecen las relaciones relevantes entre especie y ambiente en cuanto a requerimiento y comportamiento definida por la ecuación (2), la metodología se valida a través de la recopilación de datos tanto de estudios anteriores como de muestreos in situ. Para efectos de cálculo se aplica un modelo de cálculo en plani1la EXCEL, en el que se incorporaron al modelo las ecuaciones de cálculo, parámetros y variables ambientales relevantes en el cultivo de los recursos a evaluar. De este modo, se aplica un modelo de simulación que considera las variables para efectos de predicción, basándose en la metodología de cálculo propuesta y los valores mínimos, medios y máximos de oxígeno para profundidades de 0 y 10 m, extraídos de los distritos construidos.

\section{Evaluación multi-criterio y multi-objetivo}

Para determinar la aptitud para cultivo, se realiza una evaluación multi-criterio, pensado como la elección entre varias alternativas basándose en la combinación de una serie de criterios en función de un objetivo específico. Mediante una evaluación multicriterio las imágenes de los criterios que definen la idoneidad, son combinadas para generar un mapa de aptitud, a partir del cual se realizará la selección final. En este estudio se aplica el método de combinación lineal pesada (Voogd, 1983).

Primero se deben evaluar, normalizar y asignar los pesos relativos de los factores, para tal efecto se requiere que las personas comprometidas en la toma de decisiones hagan un juicio sobre la importancia relativa de combinaciones pareadas entre los criterios de selección. Después de obtener los pesos, se incorporan las restricciones, se valorizan las soluciones y se optimizan los resultados. La información de los criterios de selección es valorizada, sustentándose en una serie de requerimientos ambientales (ver ejemplo Tabla 1). Como resultado de este análisis se generan imágenes digitales donde se muestra el índice de aptitud para cada distrito (Fig. $4)$, este índice es estimado con la ecuación metodológica (1).

Para ayudar en el proceso de toma de decisión sobre qué especie cultivar y dónde, se realiza un análisis de distribución de sitios para multi-objetivos con el SIG IDRISI. Las imágenes de índices de aptitud para los objetivos de cultivo de salmónidos y cultivo de ostra japonesa, se clasifican y analizan para obtener como resultado un mapa que muestra con color rojo las celdas asignadas al primer objetivo y con color azul aquellas asignadas al segundo (Fig. 5).

\section{RESULTADOS}

\section{Determinación de distritos de aptitud para cultivos acuícolas}

Como resultado de los procedimientos descritos anteriormente se han obtenido imágenes digitales de distritos de aptitud (Figs. 3, 4 y 5) que tienen en sus bases de datos la información de todos los planos de información digital de base y adicionales generadas con los procesos del SIG.

Esto facilita la determinación de un sitio apto para cultivo, es decir, la probabilidad de éxito y potencial de cultivo, ya que del análisis y manipula- 


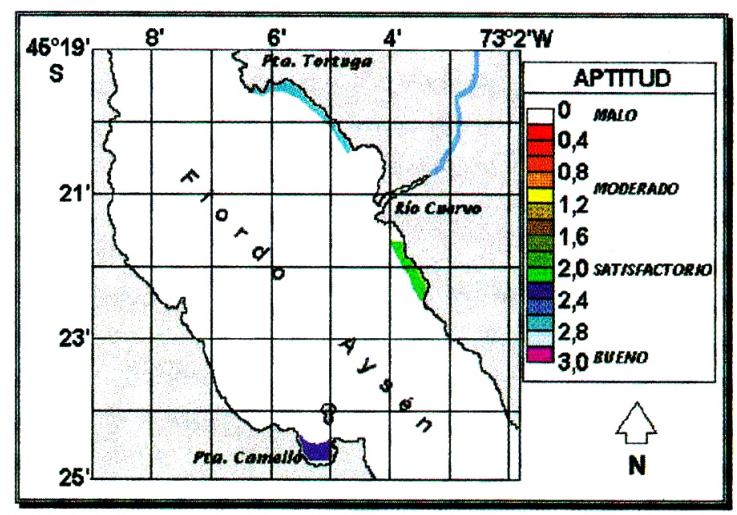

Figura 4. Mapa que muestra la aptitud de distritos para cultivo de salmónidos en balsas-jaulas.

Figure 4. Suitability for salmonid cage culture.

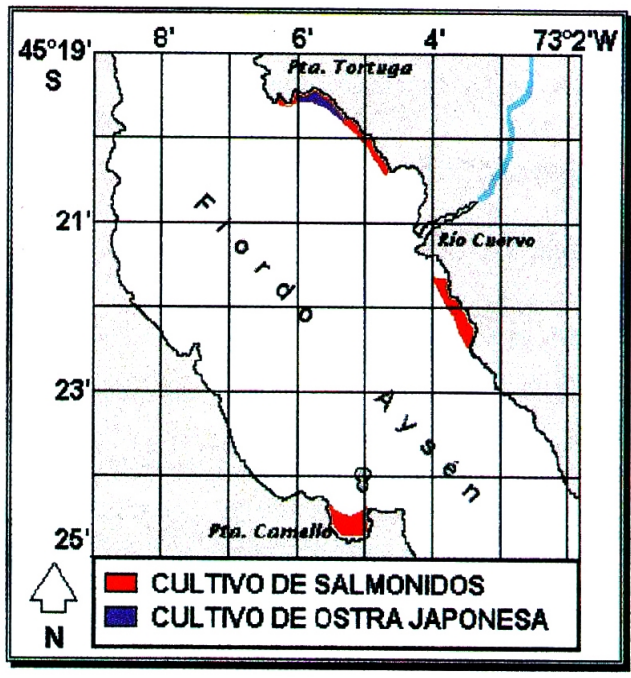

Figura 5. Mapa que propone distritos para cultivos específicos.

Figure 5. Map of aquaculture site selection.

Tabla 1. Valoración de los principales criterios de selección de distritos aptos para cultivo de salmónidos en balsas jaulas.

Table 1. Valuation of the main criteria used in salmonid cage culture site selection.

\begin{tabular}{|c|c|c|c|c|c|}
\hline Criterio & Valor & Criterio & Valor & Criterio & Valor \\
\hline Temperatura $\left({ }^{\circ} \mathrm{C}\right)$ & & Batimetría (m) & & Presencia depredadores & \\
\hline 0 a 5 & 0 & 0 a 10 & 0 & Muy Abundante & 0 \\
\hline 5 a 10 & 2 & 10 a 20 & 2 & Abundante & 1 \\
\hline 10 a 15 & 3 & 20 a 40 & 3 & Escasa & 2 \\
\hline 15 a 18 & 2 & 40 a 50 & 2 & Rara & 3 \\
\hline Más de 18 & 0 & Más de 50 & 0 & Presencia especie & \\
\hline Salinidad (\%o) & & Corrientes $(\mathrm{m} / \mathrm{s})$ & & No & 0 \\
\hline 0 a 15 & 0 & 0 a 0,1 & 0 & Sí & 3 \\
\hline 15 a 18 & 1 & 0,1 a 0,5 & 3 & Contaminación & \\
\hline 18 a 24 & 2 & 0,5 a 1 & 2 & Sí & 0 \\
\hline 24 a 35 & 3 & Más de 1 & 0 & No & 3 \\
\hline Más de 35 & 0 & Altura de ola $(m)$ & & Accesibilidad & \\
\hline Oxígeno disuelto $(\mathrm{mg} / \mathrm{l})$ & & 0 a 1,5 & 3 & Malo & 0 \\
\hline 0 a 6 & 0 & 1,5 a 2 & 2 & Deficiente & 1 \\
\hline Más de 6 & 3 & Más de 2 & 0 & Aceptable & 2 \\
\hline & & & & Bueno & 3 \\
\hline
\end{tabular}

Elaborado por los autores en base a Chen (1979), Beveridge (1987), Laird y Needham (1988), Bromage y Sheperd (1988). 
ción de las bases de datos se obtiene una imagen de síntesis final (imagen de distritos de aptitud). De acuerdo a la ecuación metodológica (1) la base de datos asociada a cada distrito o polígono de la imagen de distritos de aptitud (obtenida por superposición de las imágenes digitales de los criterios ambientales considerando los requerimientos específicos), contiene la información de los criterios de selección.

Para mostrar el proceso final, la Tabla 2 muestra algunos campos y registros de la base de datos de las imágenes de distritos de aptitud para cultivo de salmónidos en balsas jaulas. El ítem "Valor_Ai" contiene la ponderación definida por la ecuación (1), el ítem "Aptitud" define la clasificación del índice de aptitud expresado en el mapa de la Figura 4 que grafica el ejemplo seguido en este estudio. Se muestran los valores de algunos criterios ambientales como temperatura (Valor_T $\mathrm{T}^{\mathrm{o}}$ ), oxígeno (Valor_Od) y salinidad (Valor_S\%o).

Se pueden obtener valores límites de la aptitud para cultivo de 0 a 3 , siendo 0 donde los valores de los criterios en un distrito o polígono es 0 , y 3 donde los valores de los criterios en un distrito son 3 . Se definen los rangos: no aptitud (NA) los polígonos con valor 0 , aptitud baja $(\mathrm{AB})$ aquellos con valor 1 , aptitud media (AM) los con valor 2, y aptitud alta (AA) los polígonos con valor 3 .

Sin embargo, esta evaluación puede ser complementada con características cualitativas y cuantitativas que el SIG puede entregar desde la base de datos que reúne todos los factores incorporados en la metodología. Así, un distrito clasificado de aptitud media puede tener las siguientes características: temperatura mínima de $10,2^{\circ} \mathrm{C}$, media de $14,5^{\circ} \mathrm{C}$ y máxima de $16,8^{\circ} \mathrm{C}$; oxígeno disuelto mínimo de 6,5 $\mathrm{mg} / \mathrm{l}$ y medio de $7,8 \mathrm{mg} / \mathrm{l}$; profundidad media de 36 $\mathrm{m}$; velocidad media de corriente de 2,6 m/s; abundante presencia de depredadores; y accesibilidad deficiente.

\section{CONSIDERACIONES FINALES}

El método propuesto se sustenta en un carácter integral de tratamiento de la aptitud para cultivos acuícolas. Es integral, tanto en los parámetros y variables consideradas, como en la forma sistémica de asociarlos.

El método está diseñado para ser ejecutado en un Sistema de Información Geográfica, aunque puede ser realizado en forma no automatizada. Las razones de ello son la gran cantidad de información incluida en los procedimientos que no debe extraviarse en ningún momento del desarrollo de la metodología, la exactitud y fiabilidad que los SIG poseen al momento de procesar y manipular la información, y la rapidez con que estas herramientas informáticas realizan cada paso de la metodología una vez ingresada la información al SIG.

No obstante lo anterior, la confiabilidad y eficacia de los resultados obtenidos dependerá de cuatro factores a considerar, que en orden de preponderancia son: la certeza de los datos ingresados; la competencia del equipo de investigación en cuanto a su capacitación en el manejo de SIG y experiencia en estudios de evaluación pesquera; lo acucioso del contexto organizativo del SIG, que a su vez depende en gran medida del tipo de software y de la capacidades y prestezas del hardware; y las personas que emitan juicios sobre la importancia relativa de combinaciones pareadas entre los criterios de selección.

Lo fidedigno de los datos va a depender de la fuente de éstos. Los mapas topográficos pueden ser obtenidos de imágenes de satélites de alta resolución (SPOT, LANDSAT) reemplazando así el uso de perfiles topográficos (Barbieri y Silva, 1997). Los datos batimétricos suelen ser escasos y requieren de una detallada investigación; una vez obtenidos, de cualquier tipo de fuente, los datos digitalizados pasan a ser un recurso valioso y permanente. Los datos de protección y exposición del lugar pueden ser

Tabla 2. Base de datos del layer de aptitud para cultivo. Table 2. Suitabity of the suitability culture layer.

\begin{tabular}{|ccccccc|}
\hline Area (m2) & Aptitud_Id & Valor_T & Valor_Od & Valor_S\%o & Valor_Ai & Aptitud \\
\hline 33,3 & 3.000 & 2,4 & 2,6 & 1,9 & 2,5 & AA \\
29,7 & 2.000 & 2,2 & 2,5 & 1,8 & 1,9 & AM \\
26,4 & 3.000 & 2,4 & 2,2 & 1,9 & 2,1 & AA \\
\hline
\end{tabular}


obtenidos a partir de imágenes de satélites de radar (ERS-1, RADARSAT), los que correctamente integrados con los datos meteorológicos de largo plazo pueden producir un importante plano de información en el SIG (Barbieri y Silva, 1997). Las corrientes y la calidad del agua van a requerir de investigación de campo que se traducen en datos puntuales, esta información requiere de procesos de interpolación que considera supuestos y limitantes de acuerdo a la técnica de interpolación utilizada, además se puede monitorear la condición ambiental superficial a través de imágenes de temperatura del mar obtenidas del sensor AVHRR a bordo de satélites NOAA e imágenes de concentración de clorofila obtenidas del sensor SeaWifs a bordo del satélite SeaStar.

Finalmente, a modo de recomendación, se sugiere implementar en Chile un Sistema de Información Espacial de Distritos de Aptitud Acuícola sustentado en un SIG a nivel nacional, regional, provincial o en pequeñas unidades administrativas o ecológicas. Cabe señalar, que este enfoque ha sido empleado en Chile por la Subsecretaría de Pesca, la Universidad de Chile, el Instituto de Fomento Pesquero y la Universidad Católica de Valpraíso (Silva, 1998). Este sistema sirve como instrumento de regulación para definir el uso adecuado de concesiones marítimas, y como instrumento de gestión para definir la planificación de la expansión de las actividades acuícolas y de esta forma reducir los riesgos de malas instalaciones.

\section{REFERENCIAS BIBLIOGRÁFICAS}

Barbieri, M.A. y C. Silva. 1997. Zonas de repoblamiento de recursos bentónicos en la región de Valparaíso, Chile, a través de imágenes ERS-1 y SPOT. In: T.D. Guyenne (Ed.) Uso y Aplicaciones de ERS-1 en América Latina. ESA Publications Division, Noordwijk: 231-235.

Beveridge, M.C.M. 1987. Cage Aquaculture. Fishing News Books Ltd., England. 352 p.

Bromage, N. y J. Sheperd. 1988. Fish, their requirements and site evaluation. In: J. Sheperd and N. Bromage (Eds.), Intensive Fish Farming. BSP Professional Books, Oxford: 17-48.

Chen, F.Y. 1979. Progress and problems of net cage culture of grouper in Singapoure. Journal World Mariculture Society 10: 260-274.

Laird, L.M. y J. Needham. 1988. Salmon and Trout Farming. Ellis Hoorwood Ltd., England. 148 p.

Meaden, G.J. y J.M. Kapetsky. 1992. Los sistemas de información geográfica y la telepercepción en la pesca continental y la acuicultura. Documento técnico de pesca N³18 Roma, FAO. 266 p.

Meaden, G.J. y T. Do Chi. 1996. Geographical information systems: aplications to marine fisheries. FAO Fisheries Technical Paper. No 356. $335 \mathrm{p}$.

Silva, C. 1998. Determinación de distritos de aptitud acuícola, situados en el fiordo Aysén, XI Región. Tesis para optar al título de Ingeniero Pesquero. Escuela de Ciencias del Mar, Universidad Católica de Valparaíso. 96 p.

Voogd, H. 1983. Multicriteria Evaluation for Urban and Regional Planning, Pion Ltd., London. 